\title{
Breast cancer, genetics, and age at first pregnancy
}

\author{
HENRY T LYNCH*, WILLIAM A ALBANO†, MICHAEL A LAYTON*, \\ WILLIAM J KIMBERLING +, AND JANE F LYNCH* \\ From *the Department of Preventive Medicine/Public Health, and †the Department of Surgery, \\ Creighton University School of Medicine; and $\ddagger$ the Department of Otolaryngology, Boys Town \\ Institute for Hearing and Speech Disorders in Children, Omaha, Nebraska, USA.
}

SUMMARY Hereditary breast cancer shows a distinctive natural history characterised by an earlier age of onset, excess bilaterality, vertical transmission, heterogeneous tumour associations, and $\underset{\sim}{\sim}$ improved survival when compared to its sporadic counterpart. To date, very little attention has been i

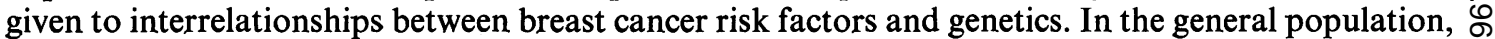
early age of first term pregnancy has been generally accepted as protective against breast cancer. ${ }^{1}$ In addition, recent findings suggest that an early age of first pregnancy may be associated with an $\overrightarrow{ }$ earlier age of breast cancer diagnosis. ${ }^{2}$

We studied the age at first pregnancy and age at onset of breast cancer among 162 females at $50 \%$ genetic risk, 72 of whom had already developed the disease. We then compared them to 154 consecutively ascertained breast cancer patients from the Creighton Cancer Center. In the hereditary subset (1) early first term pregnancy did not alter the frequency of breast cancer; (2) early age at first term pregnancy was not associated with an earlier age at cancer diagnosis; and (3) age of breast cancer onset in nulliparous females was not significantly lower than that in females having at least one term pregnancy. We speculate, therefore, that in our hereditary population, pregnancy does not influence the natural history of breast cancer in the same way that it does in the population at large.

Breast cancer incidence is determined by the interplay of myriad endogenous (race, endocrine factors, and habitus) ${ }^{3-8}$ and exogenous (geographic, socioeconomic, and dietary) events. ${ }^{19-12}$ Early age at first term pregnancy may confer protection against breast cancer. ${ }^{1}$ Within the breast cancer population there exists a significant subset in whom primary genetic factors are aetiological. ${ }^{3}$ The hereditary form is characterised by a distinct natural history when compared to its sporadic counterpart, including early age of breast cancer onset, bilaterality, vertical transmission, specific heterogeneous tumour patterns, and improved survival. ${ }^{3}$ There has been little systematic inquiry into interrelationships between breast cancer risk factors and primary genetic influences. ${ }^{13}$

Our purpose is to describe findings suggesting that in the hereditary breast cancer population, pregnancy does not influence tumour genesis in the same way that it does in the population at large.

Materials and methods

Thirty-five extended hereditary breast cancer-prone

Received for publication 14 December 1982.

Accepted for publication 3 August 1983. kindreds from our resource ${ }^{3}$ were investigated. Mendelian genetic analysis revealed 162 females at high genetic risk $(50 \%)$ for breast cancer, 72 of whom already had the disease. Within the high 0 risk population (162 patients), the percentage of $\overline{3}$ women developing breast cancer versus age at first term pregnancy was evaluated. In addition, the $\delta$ age at cancer diagnosis among 72 affected members of the high risk subset was analysed. These data 을 were compared with the age of breast cancer $D$ diagnosis versus the age at first term pregnancy in 154 consecutively ascertained breast cancer patients o seen at the Creighton Cancer Center.

Best fit lines were constructed via linear regression $N$ analysis and examined for statistical significance $\underset{\omega}{\mathrm{W}}$ with the $t$ test. Patients from the three groups were of similar socioeconomic and educational 0 backgrounds and the majority came from rural $\mathbb{\mathscr { D }}$ midwestern families of northern European $\stackrel{\oplus}{\rightarrow}$ background.

\section{Results}

Within the hereditary subset, early age at first term pregnancy did not demonstrate a 'protective' effect, 
that is, the frequency of females affected as a function of age at first pregnancy did not show a significant positive correlation $(t=1 \cdot 1$, NS) against developing the disease (fig 1).

Among the females from the hereditary population who eventually developed the disease, early age at first term pregnancy was not significantly correlated with an earlier onset of breast cancer $(t=1 \cdot 6$, NS), whereas those from the consecutive series did show a significant earlier age at diagnosis with an earlier age at first pregnancy (figs 2 and 3 ).

Age at diagnosis of breast cancer in the hereditary subset was not significantly different in parous than in nulliparous females $(t=1 \cdot 17, \mathrm{NS})$.

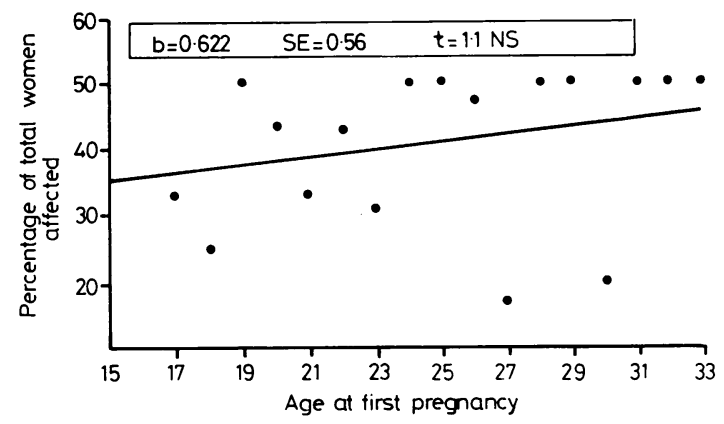

FIG 1 Percentage of females affected as a function of age at first pregnancy in the Creighton Hereditary. Breast Cancer Fainily Resource.

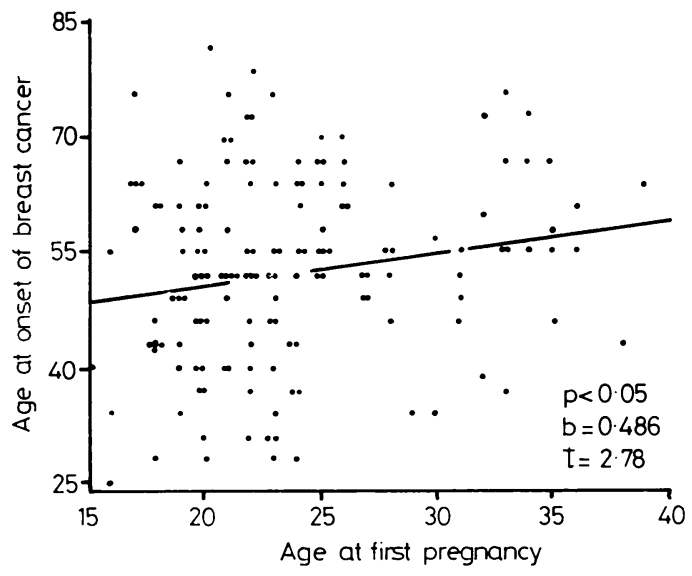

FIG 2 Age of diagnosis of breast cancer as a function of age at first term pregnancy in our consecutively ascertained series.

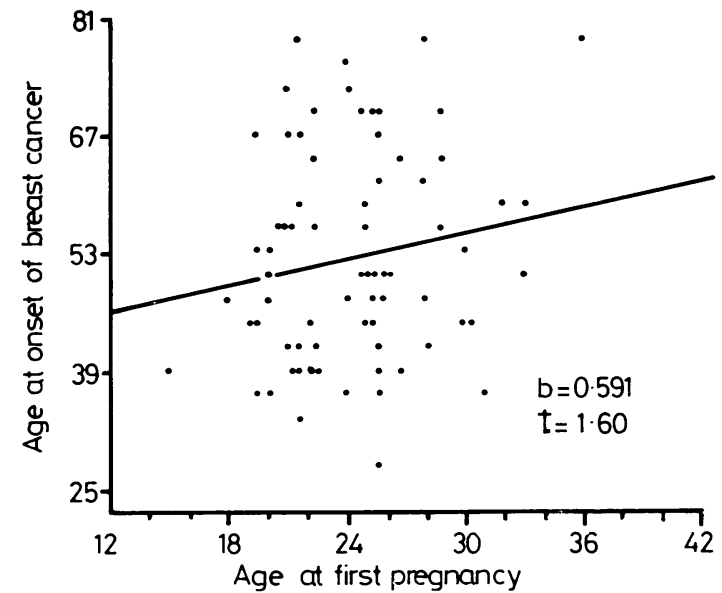

FIG 3 Age of diagnosis of breast cancer as a function of age at first term pregnancy in the hereditary subset.

\section{Discussion}

Our results, while based upon a limited sample, illustrate significant differences between hereditary breast cancer and breast cancer in the population at large with regard to the effects of pregnancy. Firstly, the generally accepted dictum of an early first term pregnancy providing a protective effect against the development of breast cancer may not be applicable to the hereditary population. Secondly, although Woods et $a l^{2}$ reported an earlier age at breast cancer diagnosis with an earlier age of first term pregnancy and an earlier age of diagnosis in nulliparous than in parous females, these relationships were not found in the hereditary population. These observations together demonstrate (1) more credence to our hypothesis of distinct biological differences between hereditary and sporadic forms of breast cancer; and (2) pregnancy does not influence tumour genesis among genetically high risk females in the same way that it does in the sporadic population.

The aetiological significance of our observation remains elusive. Given the hormonal influences of pregnancy, ${ }^{13}$ it is possible that previously observed differences in oestrogen profile in the hereditary form may harbour clues to explain these differences. ${ }^{14} 15$ Specifically, one might speculate that the different steroid patterns predispose to tumour genesis in the hereditary population irrespective of pregnancy status, whereas the fluctuating levels of oestrogens in the sporadic population during pregnancy influence tumour genesis to a high degree. It would therefore be prudent to give high priority to further studies 
of an endocrine hypothesis for elucidation of hereditary breast cancer aetiology.

It is of interest that at the animal level, using the rat as a model, chemically induced tumours provided an interesting parallel to the above findings in humans. Specifically, previous pregnancy rendered rat mammary tissue less susceptible to chemical carcinogenesis. $^{16}$ However, when the pregnancy followed exposure to the carcinogen, the interval between exposure and the presentation of tumours was shortened. ${ }^{17} 18$ This seems to parallel findings in the sporadic population quite well. ${ }^{12}$ It would be of further interest to investigate these same parameters in a strain endogenously predisposed to breast cancer.

We believe that our observations should be interpreted cautiously pending their assessment in a larger number of well documented kindreds. In addition, we believe that future studies should take into consideration heterogeneous forms of this disease, such as site specific hereditary breast cancer, ${ }^{19}$ breast cancer in combination with ovarian cancer, ${ }^{20}$ and the SBLA syndrome. ${ }^{21}$ In conclusion, early age at first term pregnancy, or pregnancy status itself, should not be considered protective among high risk relatives from hereditary breast cancer kindreds.

\section{References}

1 MacMahon B, Cole P, Brown J. Etiology of human breast cancer: a review. J Natl Cancer Inst 1973;50:21-42.

2 Woods KL, Smith SR, Morrison JM. Parity and breast cancer: evidence of a dual effect. $\mathrm{Br}$ Med J 1980;281: 419-21.

3 Lynch HT. Genetics and breast cancer. New York: Van Nostrand Reinhold, 1981.

4 Bertini B, Ber A, Posener LN, Zelikson-Singer S. Ethnic and constitutional differences and their relation to breast diseases in Israel: educational and socioeconomic status. Br J Cancer $1971 ; 25: 428-40$.

5 Kovi J, Heshmat MY. Incidence of cancer in negroes in Washington DC and selected African cities. Am J Epidemiol 1971;96:401-13.

6 Lemon HM. Endocrine influences on human mammary cancer formation: a critique. Cancer 1969;23:781-90.
7 Bulbrook RD, Hayward JL, Spicer CC. Relation between urinary androgen, corticoid excretion, and subsequent breast cancer. Lancet 1971 ;ii:395-8.

8 DeWaard F. The epidemiology of breast cancer: review and prospects. Int J Cancer 1969;4:577-86.

9 Doll R, Muir C, Waterhouse J. Cancer incidence in five continents. Vol 2. International Union Against Cancer. Berlin: Springer-Verlag, 1970.

10 Armstrong B, Doll R. Environmental factors, cancer incidence, and mortality in different countries, with special reference to dietary practices. Int $J$ Cancer 1975;15:617-31.

11 Rosenberg L, Slone D, Shapiro S, et al. Breast cancer and alcoholic beverage consumption. Lancet 1982;i:267-71

12 Lilienfeld AM, Coombs J, Bross IDJ, Chamberlain A Marital and reproductive experience in a communitywide epidemiological study of breast cancer. Johns Hopkins Med J 1975;136:157.

13 Brinton LA, Hoover R, Fraumeni JF. Interaction of familial and hormonal risk factors for breast cancer. J Natl Cancer Inst 1982;69:817-22.

14 Fishman J, Fukushima D, O'Connor J, et al. Plasma hormone profiles of young women at risk for familial breast cancer. Cancer Res 1978;48:4006-11.

15 Fishman J, Fukushima D, O'Connor J, Lynch HT. Low urinary estrogen glucuronides in women at risk for familial breast cancer. Science 1979;204:1089-91.

16 Moon RC. Relationship between previous reproductive history and chemically induced mammary cancer in rats. Int J Cancer 1969;23:312.

17 Dao TL, Sunderland H. Mammary carcinogenesis by 3-methycholanthrene. I. Hormonal aspects in tumor induction and growth. J Natl Cancer Inst 1959;23:567.

18 McCormick GM, Moon RC. Effect of pregnancy and lactation on growth of mammary tumors induced by 7,12 DMBA. Br J Cancer 1965;19:160.

19 Lynch HT, Krush AJ, Lemon HM, Kaplan AR, Condit PT, Bottomley RH. Tumor variation in families with breast cancer. JAMA 1972;222:1631-5.

20 Lynch HT, Harris RE, Guirgis HA, Maloney K, Carmody L, Lynch JF. Familial association of breast/ ovarian cancer. Cancer $1978 ; 41: 1543-8$.

21 Lynch HT, Mulcahy GM, Harris RE, Guirgis HA, Lynch JF. Genetic and pathologic findings in a kindred with hereditary sarcoma, breast cancer, brain tumors, leukemia, lung, laryngeal, and adrenal cortical carcinoma. Cancer 1978;41:2055-64.

Correspondence and requests for reprints to Professor H T Lynch, Department of Preventive Medicine and Public Health, Creighton University School of Medicine, Omaha, Nebraska 68178, USA. 